\title{
Evaluation of Novel Anticaries Adhesive in a Secondary Caries Animal Model
}

\author{
Tianmu Wua, $\mathrm{u}^{\mathrm{a}}$ Bolei $\mathrm{Li}^{\mathrm{a}} \mathrm{b}$ Xuedong Zhou ${ }^{\mathrm{a}, \mathrm{b}}$ Yao Hu $\mathrm{Hu}^{\mathrm{a}, \mathrm{b}}$ Huajin Zhang ${ }^{\mathrm{c}}$ \\ Yingkang Huang ${ }^{a}$ Hockin H.K. Xu ${ }^{d}$ Qiang Guo ${ }^{a}$ Mingyun Li $^{a}$ Mingye Feng ${ }^{a}$ \\ Xian Peng $^{a}$ Michael D Weir ${ }^{d}$ Lei Cheng ${ }^{a, b}$ Biao Ren ${ }^{a}$ \\ ${ }^{a}$ State Key Laboratory of Oral Diseases, b Department of Operative Dentistry and Endodontics, West China Hospital \\ of Stomatology and 'Key Laboratory of Drug Targeting and Drug Delivery Systems, West China School of Pharmacy, \\ Sichuan University, Chengdu, China; ${ }^{d}$ Biomaterials and Tissue Engineering Division, Department of Endodontics, \\ Periodontics and Prosthodontics, University of Maryland Dental School, Baltimore, MD, USA
}

\section{Keywords}

Antibacterial material · Bonding system .

Dimethylaminododecyl methacrylate $\cdot$ Keyes animal model $\cdot$ Micro-CT $\cdot$ Tooth restoration

\begin{abstract}
We investigated the anticaries properties of an adhesive containing dimethylaminododecyl methacrylate (DMADDM) in vivo via a secondary caries animal model. Cavities were prepared in the maxillary first molars of Wistar rats. DMADDM-containing adhesives were applied on one side and commercial adhesives on the opposite side as a control. After a 3-week feeding period to induce secondary caries, the molars were harvested for the evaluation of the secondary caries. Lesion depth (LD) and mineral loss (ML) were measured via a micro-CT method, and a modified Keyes scoring method yielded scores for the caries lesions. Statistical analysis was divided into 2 parts: a correlation analysis between 2 evaluations with one-way ANOVA and a least-significant differences (LSD) test, and an evaluation of anticaries adhesives with a paired samples $t$ test. The results showed that:
\end{abstract}

\section{KARGER}

E-Mail karger@karger.com www.karger.com/cre

\section{The Author(s) \\ Published by S. Karger AG, Basel \\ Karger \\ Open access}

This article is licensed under the Creative Commons AttributionNonCommercial-NoDerivatives 4.0 International License (CC BYNC-ND) (http://www.karger.com/Services/OpenAccessLicense). Usage and distribution for commercial purposes as well as any distribution of modified material requires written permission.
(1) secondary caries was successfully produced in rats; (2) there was a correlation between the modified Keyes scoring method and micro-CT in the evaluation of the secondary caries; (3) the adhesive containing DMADDM significantly reduced both LD and ML (according to micro-CT), and also lowered the scores (based on the modified Keyes scoring method). This suggests that the novel DMADDM adhesive could perform an anticaries function in vivo via the secondary caries animal model which was also developed and testified in research. Secondary caries is one of the major reasons leading to the failure of caries restoration treatment. As a solution, anticaries adhesives perform well in biofilm inhibition in vitro. However, the lack of secondary caries animal models limits the evaluation of anticaries adhesives in vivo.

(c) 2017 The Author(s)

Published by S. Karger AG, Basel

Secondary caries is one of the main reasons for restoration failure with a heavy economic burden [Sakaguchi, 2005; Kasraei et al., 2017]. While many factors facilitate the development of secondary caries, oral bacteria and acid production are the initiators of dental caries [Mjor 
and Toffenetti, 2000; Takahashi and Nyvad, 2011]. Efforts have been made to develop antibacterial dental materials to combat bacteria and secondary caries. Several bioactive agents have been synthesized and incorporated into dental materials [Cheng et al., 2012, 2013; Huang et al., 2012; Shi et al., 2012], including various quaternary ammonium monomers (QAMs) that demonstrate favorable antibacterial properties.

QAMs exert a contact-killing mechanism and have been shown to effectively kill bacteria and inhibit biofilm growth, not only on single-species biofilms, but also on multispecies biofilms [Zhang et al., 2015; Wang et al., 2016]. In addition, the antimicrobial properties are considered to be related to chain lengths and charge densities [Zhou et al., 2013, 2014]. 12-Methacryloyloxydodecylpyridinium bromide (MDPB) has been incorporated into dental resins for a strong antimicrobial function [Imazato et al., 1994, 2003]. Anticaries adhesives have been developed by supplementing with various QAMs, including methacryloxylethyl cetyl dimethyl ammonium chloride (DMAE-CB) [Li et al., 2009], quaternary ammonium dimethacrylate (QADM) [Cheng et al., 2012], and, more recently, dimethylaminododecyl methacrylate (DMADDM) [Cheng et al., 2013]. DMADDM provides potent antibacterial activity without compromising the physical properties of the adhesive. However, the lack of an animal model for testing secondary caries has limited the investigation of the efficacy of secondary caries in vivo.

There is no existing animal model for secondary caries. An important animal model for the study of caries study was originally investigated by Keyes [1954, 1956, 1958a, b], who set a template in 2 essential aspects, i.e., caries production and caries evaluation [Stookey et al., 1995]. As a template, the Keyes model was modified into different forms to match the various demands of experiments, but without changing its outline of caries production, idea of caries evaluation, and linear estimates [Bowen et al., 1988; Bowen and Pearson, 1992]. Besides this classic method, the detection of caries by means of various instruments was also developed. Transverse microradiography has been the most widely accepted gold standard for caries detection and evaluation in vitro, but the strict requirements regarding sample preparation limit its usage [Lo et al., 2010]. Micro-CT is now considered a substitute for this gold-standard method of caries detection because of its ease of use [Lo et al., 2010; Hamba et al., 2012]. Among the multiple methods, the Keyes method for evaluation is always favored because of its simplicity and low cost. However, as a method constructed for primary caries study, the Keyes animal model without any

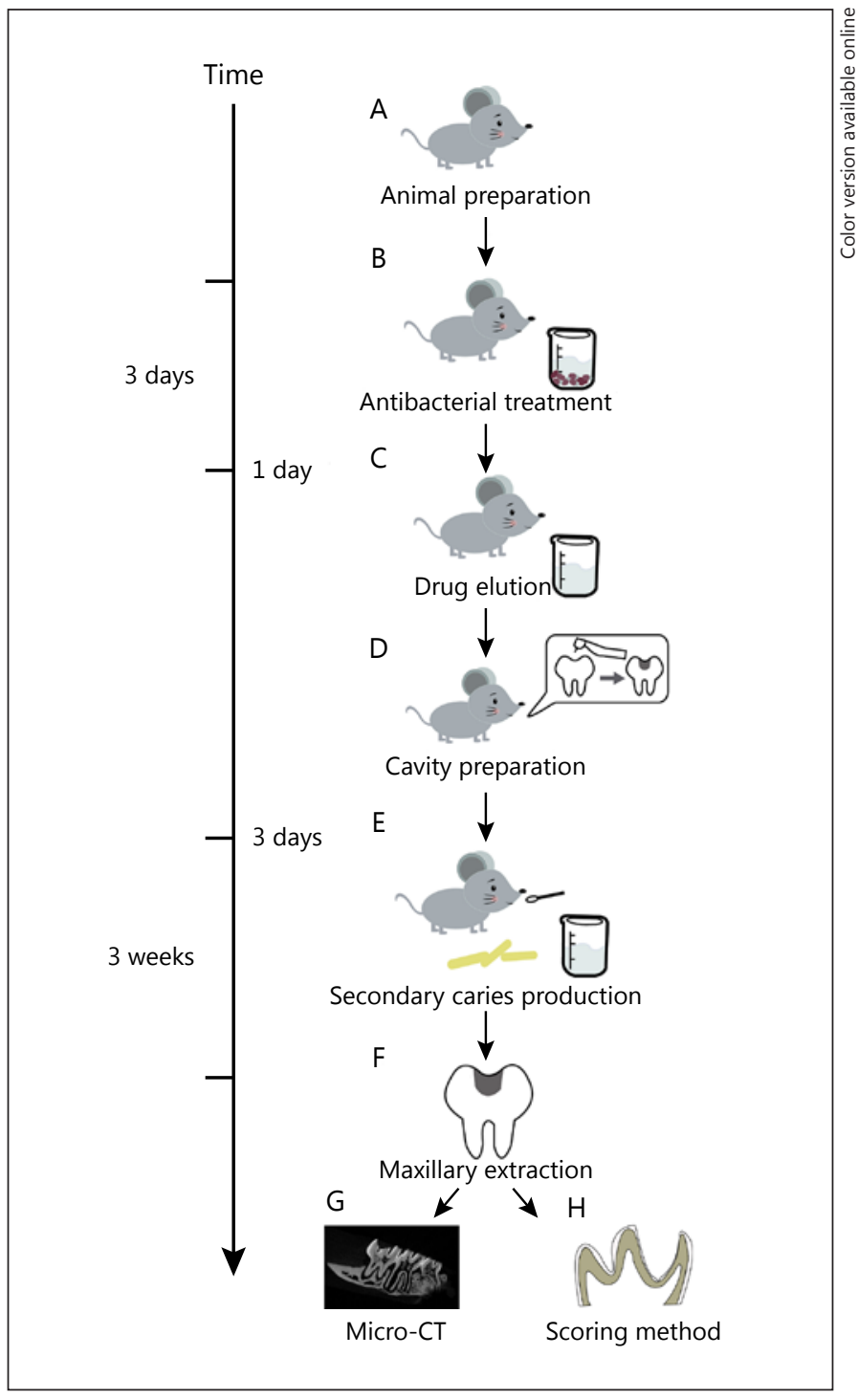

Fig. 1. Schematic of the experiments: A, animal preparation; B, antibacterial treatment (drinking water containing ampicillin for 3 days); C, drug elution (drinking common water for 1 day); $D$, cavity preparation; E, production of secondary caries (inoculating with bacteria for 3 days and feeding with cariogenic food and water for 3 weeks); F, maxillary extraction; G, micro-CT scanning evaluation; $\mathrm{H}$, evaluation by the modified Keyes caries scoring method.

modifications does not satisfy the requirements for the study of secondary caries in current research.

The objectives of this study were to modify the Keyes model for evaluating secondary caries and to explore the anticaries properties of DMADDM adhesive via the modified model in vivo. It was hypothesized that: (1) the animal model could produce secondary caries and the mod- 




Fig. 2. Evaluation by micro-CT. a Sectional view. The ROI of tissue for analysis is along the line marked by the arrow. $\mathbf{b}$ For the evaluation of the adhesive, the mineral volume (on average) of the ROI in the control and DMADDM groups is shown. The direction of the sample position ( $x$ axis) keeps pace with the red arrow in a. c Lesion depth. d Mineral loss. Data are presented as mean \pm standard deviation. ${ }^{* * *} p<0.001$.

\section{Cavity Preparation and Filling Operation}

Operations were performed on the day of drug elution (the 4th day). Rats under general anesthesia were placed in a dorsal position. Cavities were prepared in the first grooves of the maxillary first molar on both sides via an air turbine with long-necked, round bur (diameter, $0.6 \mathrm{~mm}$ ). To make a good retention form, but avoiding exposure of the dental pulp, a cavity was prepared (0.3-0.6 $\mathrm{mm}$ in depth and approx. $0.6 \mathrm{~mm}$ in diameter). Rats with accidentally exposed pulp were excluded from the study.

After cavity preparation, the rats were randomly and evenly assigned to 2 groups. Group $1(n=13)$ was filled with commercial SE Bond in the left cavity (control), and the DMADDM-containing SE Bond in the right side. Group $2(n=13)$ was filled with the left- and right-side materials being swapped. All filling operations took place according to the instructions of the SE Bond manufacturer.

\section{Secondary Caries Production}

Starting from the 5th day, a solution of Streptococcus mutans UA159 $\left(10^{8} \mathrm{CFU} / \mathrm{mL}, 0.3 \mathrm{~mL}\right)$ was scrubbed into the mouths of the rats for 3 consecutive days. Bacteria-scrubbing was performed twice each day, at a time interval of $30 \mathrm{~min}$. The rats were prevented from drinking water from the beginning of the scrubbing until 30 min after it. Simultaneously, a cariogenic diet 2000 (TrophicDiet, Trophic Animal Feed, Suzhou, China) was provided, and water was substituted with sucrose-containing water (5\% concentration). After 3 weeks, the rats were executed, and their maxillaries were obtained, with soft tissue and adhesive material being removed for better dyeing and observation.

\section{Animal Preparations}

Specific-pathogen-free male Wistar rats (Chengdu Dossy Experimental Animals Co., Ltd, China) were obtained at the age of 21 days. All rats were fed with water containing ampicillin $(1 \mathrm{~g} / \mathrm{kg}$ of concentration) for the first 3 days, and then common drinking water for another day to elute the drug [Keyes, 1960]. 

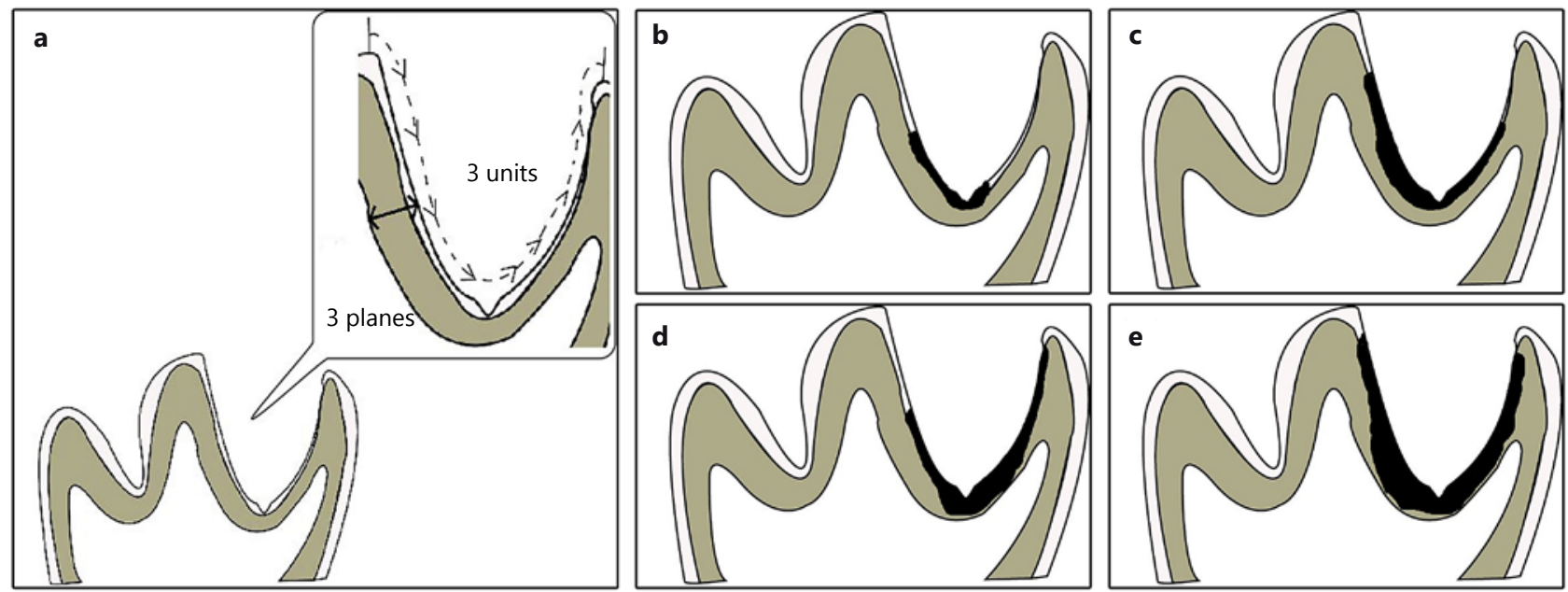

Fig. 3. Modified Keyes caries scoring method. a First, a groove was located for scoring, then the whole length along the margin of the cavity was divided equally into 3 units and the whole depth into 3 planes. b-e Black areas represent examples of scoring of secondary caries tissue. b Secondary caries involved the S-plane only, and the width occupied one-third of the whole length, i.e., 1 unit of 3. So,

\section{Micro-CT Analysis}

Specimens of maxillaries were measured by micro-CT ( $\mu$ CT 50 , SCANCO Medical AG, Brüttisellen, Switzerland). They were fastened in cylinders on a computer-controlled turntable with the lingual surface horizontal. They were scanned at medium resolution, with parameters $70 \mathrm{kVp}$ and $200 \mu \mathrm{A}$ to detect secondary caries. Each sample was rotated $360^{\circ}$ within $14.3 \mathrm{~min}$. Images were acquired and analyzed using SCANCO evaluation software v1.1.11.0 (SCANCO Medical AG). A sectional view, in which the pre-prepared cavity could be observed clearly, was chosen for each specimen. In this sectional view, a line was chosen as the region of interest (ROI) to be analyzed. This line had to pass through the border of the prepared cavity and be perpendicular to the enameldentinal junction (Fig. 2a). The mineral volume of ROI was measured by SCANCO evaluation software. The lesion depth (LD) and mineral loss (ML, due to demineralization) of the ROI were calculated to evaluate the degree of secondary caries.

Modified Keyes Scoring Method for Secondary Caries Evaluation

After the micro-CT analysis, specimens were prepared for the modified Keyes scoring method analysis. They were managed similarly to in the Keyes model. All specimens were dyed with ammonium purpurate (concentration of $0.4 \%$ ) for $12 \mathrm{~h}$, and then hemisectioned in the mesiodistal direction with a cutter $(0.1 \mathrm{~mm}$ in thickness). They were then examined and scored by means of a stereomicroscope (Leica EZ4HD, Leica Microsystems AG, Heerbrugg, Switzerland) to evaluate secondary caries in the cavities. Under the stereomicroscope, the secondary caries lesion was dyed a red color but the sound, hard tissues were not. The modified Keyes scoring method involves, by judging under a stereomicroscope, the linear extent of a lesion in 1 plane in the cavity, and recording the the score was 1, 0, 0. c Secondary caries involved the S-plane and M-plane, with widths of 2 units and 1 unit, separately, scored as 2, 1,0 . d Secondary caries involved the whole thickness, and at each plane, the width was 2 units, 1 unit, and 1 unit, scored as 2, 1, 1. e The score of secondary caries was 3,2, 2 .

depth of penetration at 3 planes (instead of the 4 planes described by Keyes [1958b]): slight (S-plane), moderate (M-plane), and extensive (X-plane). Lesions considered as being in the $\mathrm{X}$-plane means that the secondary caries has evolved beyond two-thirds of the whole depth (thickness of enamel-dentin tissue), lesions in the M-plane are those occurring in one- to-two-thirds, and those in the S-plane in less than one-third of the tooth (Fig. 3a). The width of the lesion is treated differently from the depth of penetration as in the Keyes model [Keyes, 1958b], i.e., the evaluation of secondary caries in the cavity is achieved by applying a linear estimation to a theoretically flattened-out cavity, with the whole linear length divided into 3 units at a specific plane (Fig. 3a). It should be noted that the unit should not be divided further and should always be an integer [Keyes, 1958b]. The extent of the lesion was recorded by the number of units it implicates (from 0 to 3 ) at each plane. Two sides of the sectioned half-jaw were examined and the one with greater involvement was recorded for evaluation as described by Keyes [1958b]. This is indicated in the sketches of scoring in Figure 3b-e and in the practical examples in Figure $4 a$, b.

\section{Statistical Analysis}

Statistical analysis consisted of 2 parts: a correlation analysis between 2 evaluations and the evaluation of the anticaries adhesive.

\section{Correlation Analysis}

Secondary caries were evaluated by 2 methods separately to reveal their correlation. Samples from 26 rats were divided into 3 groups (the $\mathrm{S}, \mathrm{M}$, and $\mathrm{X}$ groups) according to the evaluation of the scoring method on depth $(n=52)$. For instance, a sample with a score $(2,2,0)$ would be assigned to the M group. After grouping, one-way ANOVA and the least-significant differences (LSD) test 

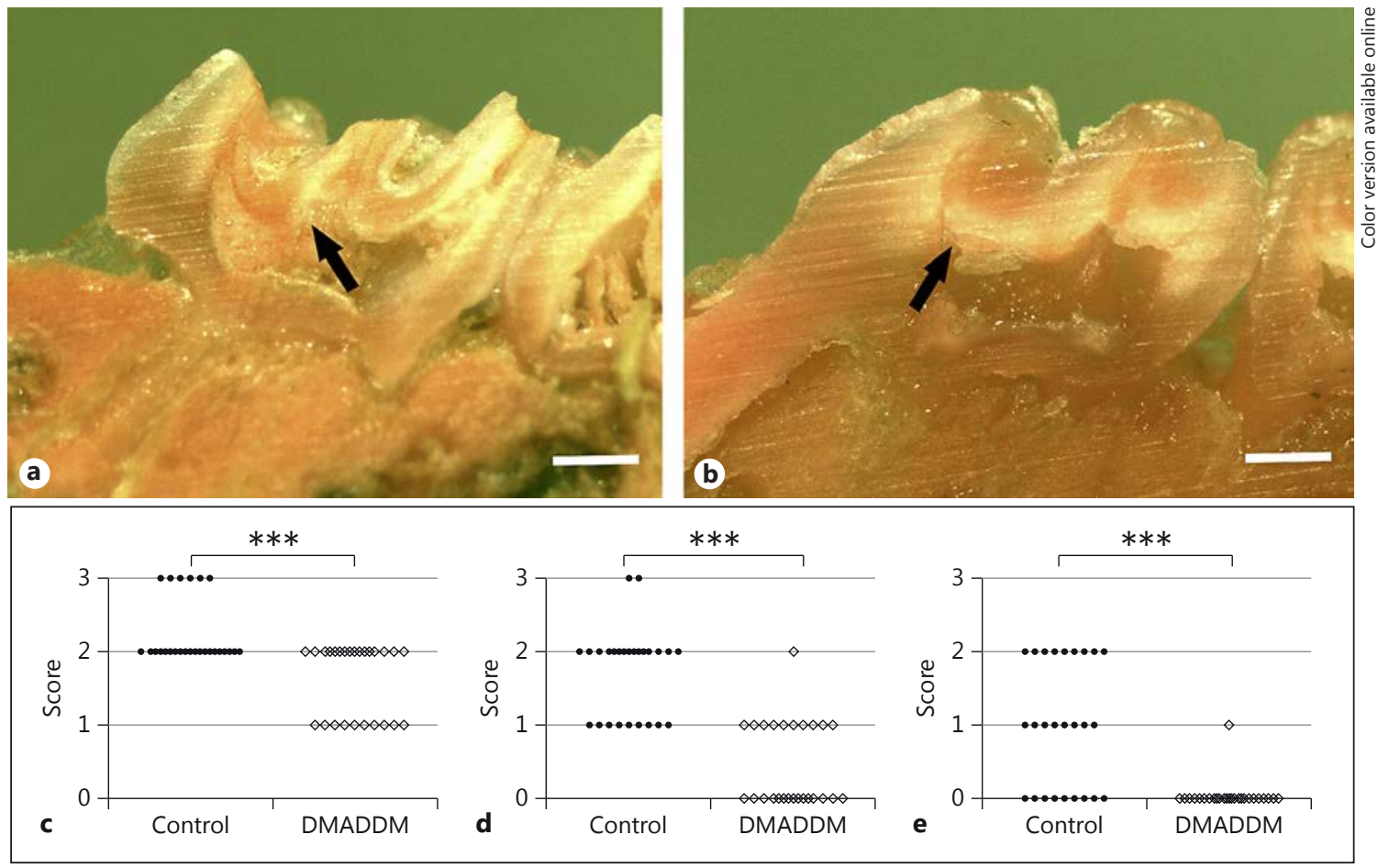

Fig. 4. Evaluation by modified Keyes caries scoring method. a, b Treated maxillary; secondary caries tissues in first grooves are dyed red (black arrows). Scale bar, $0.5 \mathrm{~mm}$. For evaluation of the adhesive, the scores of the 2 groups are illustrated separately at 3 planes: the S-plane (c), M-plane (d), and X-plane (e). Data are presented as scatter plots. ${ }^{* * *} p<0.001$

were performed to compare the LD and ML across groups. Similarly, width was divided into 4 groups based on units in the range of $0-3$ at the 3 planes separately, and was tested with the same test on their ML $(n=52)$.

Evaluation of the Anticaries Adhesive

The paired-samples $t$ test was employed in the micro-CT evaluation ( $\mathrm{LD}$ and $\mathrm{ML}$ ) to indicate the anticaries properties of the adhesive with/without DMADDM, and the Wilcoxon matchedpairs signed-ranks test was used to compare the degree of secondary caries between the 2 sides for the same objective.

The level of significance was set as $p=0.05$. Statistical analysis was conducted using SPSS v16.0 (SPSS Inc. Chicago, IL, USA).

\section{Results}

\section{Secondary Caries on Molars}

The mineral volume of the samples was measured by micro-CT (Fig. 2b). The fact that the mineral volume in the ROI was not always $100 \%$ revealed varying degrees of ML due to secondary caries. Meanwhile, red areas in the grooves could be seen on almost all samples under the stereomicroscope, indicating secondary caries. This was according to the general phenomenon that secondary caries tissue would be dyed red by ammonium purpurate, while healthy tissue would not. Representative images of the dyed maxillaries are shown in Figure $4 a$, b.

\section{Correlation Analysis}

Regarding the LD grade, compared with the S-plane, the LD and ML were higher in the M-plane, and highest in the X-plane (Fig. 5a, b). In the evaluation of the width grade, ML increased when the unit went higher at each plane (Fig. $5 c-e)$. There was no "0" score at the S-plane (Fig. 5c) and no "3" score at the X-plane (Fig. 5e).

\section{Evaluation of the Anticaries Adhesive}

Intuitively, the line of mineral volume of the DMADDM group was located above the line of the control group from beginning to end (Fig. 2b). This also indicates that the ML (the area surrounded by: line of data, $y$ axis, and $y=100$ ) in the control group was greater than that in the DMADDM group, and the maximum LD (maximum value of $x$ on the line) in the control group was greater than that in the DMADDM group (Fig. 2b). It was 


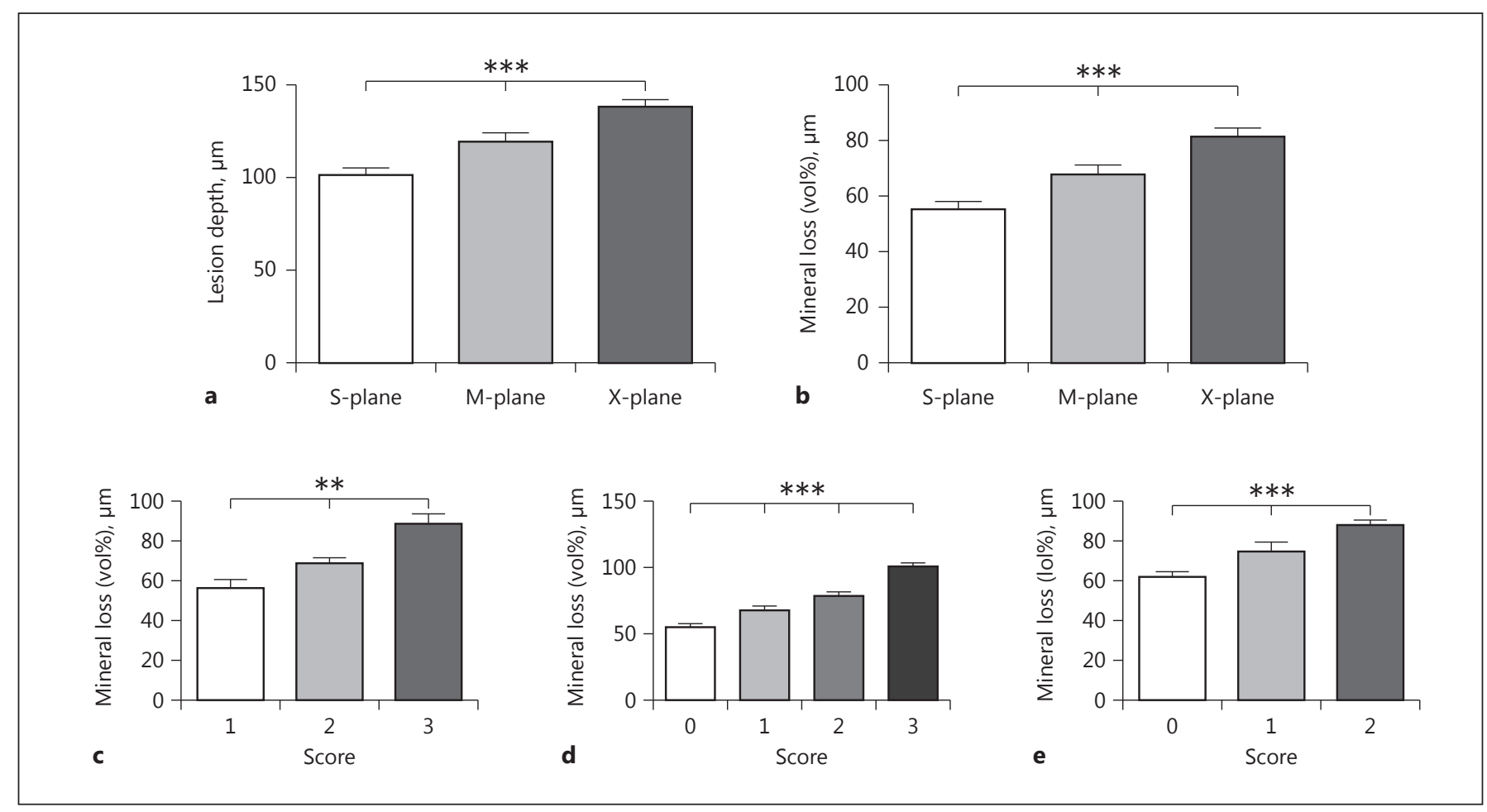

Fig. 5. Correlation analysis. The modified Keyes caries scoring method was evaluated by micro-CT on the grade of lesion depth (a) and mineral loss (b), and width grade at the S-plane (c), M-plane (d), and X-plane (e). Data are presented as mean \pm standard deviation. ${ }^{* *} p<0.01,{ }^{* * *} p<0.001$.

also shown directly that the LD and ML in the control group were significantly greater than in the DMADDM group (LD and ML: $p<0.0001$ ) (Fig. 2c, d).

The scoring method showed greater values on the non-DMADDM side than on the DMADDM side at each plane $(p<0.0001)$ (Fig. $4 c-e)$. At the S-plane, most of the values on the non-DMADDM side had scores of " 2 " and " 3 ," while scores were scattered among " 1 " and " 2 " on the DMADDM side (Fig. 4c). At the M-plane, scores were dispersed at " 1 " and " 2 " mostly on the non-DMADDM side, but at "0" and "1" on the other side (Fig. 4d). An extreme phenomenon at the X-plane in the DMADDM group was that almost all scores approached " 0 ," while the scores were dispersed in " 0 ," " 1 ," and " 2 " on the nonDMADDM side (Fig. 4e).

\section{Discussion}

We report here on an in vivo animal model for evaluating secondary caries development and the caries-inhibiting effect of antibacterial dental adhesive. A rat model for secondary caries was constructed based on the animal model of Keyes [1958a, b]. This was compared with micro-CT which is regarded as a substitute gold standard to test its rationality [Lo et al., 2010]. DMADDM-containing SE Bond was chosen as the filling material to study the anticaries properties. Our research has shown that secondary caries could be produced successfully in the animal model. Meanwhile, the reliability of the modified Keyes scoring method was confirmed by micro-CT (Fig. 5). In addition, the significant difference between the control group and the DMADDM group regarding the development of secondary caries was verified via micro-CT and the modified Keyes scoring method, demonstrating the anticaries properties of DMADDM in vivo.

The antibiofilm effect of DMADDM has been indicated in many previous studies in vitro. Furthermore, animal experiments were also performed to test the biological safety of DMADDM. When incorporated into composites and adhesives to investigate pulp reaction, DMADDM exhibited milder pulp inflammation and much greater tertiary dentin formation than composite and adhesive only [Li et al., 2014]. 
Animal models of caries have existed for nearly a century since McCollum et al. [1922] first utilized this method for caries research. However, it is a challenge to construct animal models for secondary caries, because of the extremely small teeth of the rats and the lack of proper evaluation methods. In our preliminary experiments, problems due to the tiny teeth were overcome, and the Keyes caries animal model was modified to construct the secondary caries animal model for this study. The Keyes animal model has been modified to meet the requirements of specific experiments in previous studies. For example, it has been modified to study the anticaries properties of fluoride and emodin [Larson, 1981; Culp et al., 2005; Swain and Xue, 2009; Xu et al., 2014]. In this study, it was modified for secondary caries and tested via microCT: (1) secondary caries was successfully produced via the modified Keyes animal model (Fig. 2b), and (2) it was found reliable to estimate second caries by the modified Keyes scoring method (Fig. 4a, b).

Dental caries is a disease with the result of demineralization in hard tissues; hence, the detection and evaluation of lesions should focus on the density of the hard tissues [Swain and Xue, 2009]. In the exploration of caries detection and evaluation, especially with high-tech equipment, LD and ML are 2 of the most important parameters to indicate the degree of the demineralized dental tissues [Hamba et al., 2012]. Transverse microradiography is the most widely recognized gold standard for caries detection, and LD and ML could be obtained by this method directly [Hamba et al., 2012; Lippert and Lynch, 2014]. However, its complex processes of sample preparation and destructiveness on samples limit its applications on rat teeth. Instead, as a nondestructive technique, micro-CT has been demonstrated to be valid in caries detection, can provide LD/ML values for evaluating demineralized tissue [Stock et al., 2002; Taylor et al., 2010; Kamburoglu et al., 2011; Ozkan et al., 2015], and is considered a substitute for transverse microradiography in studies on caries in vitro [Lo et al., 2010; Hamba et al., 2012]. Despite several advantages of micro-CT, the high cost and long scanning time are the disadvantages. Therefore, the Keyes scoring method is still widely used for analyzing the primary dental caries in animal models and was modified for secondary caries in this study along with a demonstration of micro-CT. In addition, secondary caries lesions are thought to be associated with spaces between the tooth tissue and the restorations; this is why a line was chosen which located the side wall of the cavity as the ROI [Diercke et al., 2009; Nassar and Gonzalez-Cabezas, 2011].
In conclusion, this research has taken the much-needed step of finding an animal model and an evaluation method for secondary caries via the modified Keyes caries animal model and its scoring method, thereby enabling the study of secondary caries in vivo. Furthermore, the adhesive containing DMADDM was shown to have significant anticaries effects in vivo, which indicates the potential clinical inclusion of DMADDM in various dental materials in order to inhibit secondary caries.

\section{Acknowledgments}

We thank the State Key Laboratory of Oral Diseases, West China Hospital of Stomatology, West China School of Pharmacy of Sichuan University, and the University of Maryland Dental School for their support throughout the research project.

\section{Disclosure Statement}

The authors declare no conflicts of interest.

\section{Funding Sources}

This study was supported by the National Key Research and Development Program of China 2016YFC1102700 (X.Z.); the National Natural Science Foundation of China grant Nos. 81372889 (L.C.), 81600858 (B.R.), 81372890 (M.F.) and 81430011 (X.Z.); the Open Fund of State Key Laboratory of Oral Diseases (2040305193002); the Recruitment Program for Young Professionals (M.F.); the NIH R01 DE17974 (H.X.); a University of Maryland School of Dentistry bridging fund (H.X.); and a University of Maryland seed grant (H.X.).

\section{Author Contributions}

Tianmu $\mathrm{Wu}$ and Bolei Li conceived and designed the experiments; Tianmu Wu, Xuedong Zhou, Yao Hu, Huajin Zhang, Yingkang Huang, Hockin H. K. Xu, and Qiang Guo performed the experiments; Mingyun Li, Mingye Feng, Tianmu Wu, Xian Peng analyzed the data; Michael D. Weir and Lei Cheng wrote the paper; Lei Cheng and Biao Ren revised the paper.
Wu/Li/Zhou/Hu/Zhang/Huang/Xu/Guo/ Li/Feng/Peng/Weir/Cheng/Ren 


\section{References}

Bowen WH, Pearson SK: The effects of sucralose, xylitol, and sorbitol on remineralization of caries lesions in rats. J Dental Res 1992;71: 1166-1168.

Bowen WH, Pearson SK, Young DA: The effect of desalivation on coronal and root surface caries in rats. J Dent Res 1988;67:21-23.

Cheng L, Weir MD, Zhang K, Arola DD, Zhou X, $\mathrm{Xu} \mathrm{HH}$ : Dental primer and adhesive containing a new antibacterial quaternary ammonium monomer dimethylaminododecyl methacrylate. J Dentistry 2013;41:345-355.

Cheng L, Zhang K, Melo MA, Weir MD, Zhou X, $\mathrm{Xu} \mathrm{HH}$ : Anti-biofilm dentin primer with quaternary ammonium and silver nanoparticles. J Dental Res 2012;91:598-604.

Culp DJ, Quivey RQ, Bowen WH, Fallon MA, Pearson SK, Faustoferri R: A mouse caries model and evaluation of aqp5-/- knockout mice. Caries Res 2005;39:448-454.

Diercke K, Lussi A, Kersten T, Seemann R: Isolated development of inner (wall) caries like lesions in a bacterial-based in vitro model. Clin Oral Investig 2009;13:439-444.

Hamba H, Nikaido T, Sadr A, Nakashima S, Tagami J: Enamel lesion parameter correlations between polychromatic micro-CT and TMR. J Dent Res 2012;91:586-591.

Huang X, Cheng L, Exterkate RA, Liu M, Zhou X, Li J, ten Cate JM: Effect of $\mathrm{pH}$ on Galla chinensis extract's stability and anti-caries properties in vitro. Arch Oral Biol 2012;57:10931099.

Imazato S, Kinomoto Y, Tarumi H, Ebisu S, Tay FR: Antibacterial activity and bonding characteristics of an adhesive resin containing antibacterial monomer MDPB. Dent Mater 2003; 19:313-319.

Imazato S, Kuramoto A, Takahashi Y, Ebisu S, Peters MC: In vitro antibacterial effects of the dentin primer of Clearfil Protect Bond. Dent Mater 2006;22:527-532.

Imazato S, Torii M, Tsuchitani Y, Mccabe JF, Russell RR: Incorporation of bacterial inhibitor into resin composite. J Dent Res 1994;73: 1437-1443.

Kamburoglu K, Kurt H, Kolsuz E, Oztas B, Tatar I, Celik HH: Occlusal caries depth measurements obtained by five different imaging modalities. J Digit Imaging 2011;24:804-813.

Kasraei S, Shokri A, Poorolajal J, Khajeh S, Rahmani H: Comparison of cone-beam computed tomography and intraoral radiography in detection of recurrent caries under composite restorations. Braz Dent J 2017;28:85-91.
Keyes PH: Dental caries in the Syrian hamster. VI. Minimal dental caries activity in animals fed presumably cariogenic rations. J Dent Res 1954;33:830-841.

Keyes PH: Dental caries in the Syrian hamster. VII. Increased dental caries activity in animals fed whole-wheat ash diets during the odontogenic period. J Dent Res 1956;35:95-101.

Keyes PH: Dental caries in the molar teeth of rats. I. Distribution of lesions induced by high-carbohydrate low-fat diets. J Dent Res 1958a;37: 1077-1087.

Keyes PH: Dental caries in the molar teeth of rats. II. A method for diagnosing and scoring several types of lesions simultaneously. J Dent Res 1958b;37:1088-1099.

Keyes PH: The infectious and transmissible nature of experimental dental caries. Findings and implications. Arch Oral Biol 1960;1:304320.

Larson R: Merits and modification of scoring rat dental caries by Keyes' method; in Tanzer JM (ed): Proceedings of Symposium on Animal Models in Cariology. Washington, IRL Press, 1981, pp 195-204.

Li F, Chai ZG, Sun MN, Wang F, Ma S, Zhang L, Fang M, Chen JH: Anti-biofilm effect of dental adhesive with cationic monomer. J Dent Res 2009;88:372-376.

Li F, Wang P, Weir MD, Fouad AF, Xu HH: Evaluation of antibacterial and remineralizing nanocomposite and adhesive in rat tooth cavity model. Acta Biomater 2014;10:2804-2813.

Lippert F, Lynch RJ: Comparison of Knoop and Vickers surface microhardness and transverse microradiography for the study of early caries lesion formation in human and bovine enamel. Arch Oral Biol 2014;59:704-710.

Lo EC, Zhi QH, Itthagarun A: Comparing two quantitative methods for studying remineralization of artificial caries. J Dentistry 2010;38: 352-359.

McCollum EV, Simmonds N, Kinney E: The relation of nutrition to tooth development and tooth preservation. I. A preliminary study of gross maxillary and dental defects in 220 rats on defective and deficient diets. Johns Hopkins Hosp Bull 1922;33:202-215.

Mjör IA, Toffenetti F: Secondary caries: a literature review with case reports. Quintessence Int 2000;31:165-179.

Nassar HM, Gonzalez-Cabezas C: Effect of gap geometry on secondary caries wall lesion development. Caries Res 2011;45:346-352.

Özkan G, Kanli A, Başeren NM, Arslan U, Tatar I: Validation of micro-computed tomography for occlusal caries detection: an in vitro study. Braz Oral Res 2015;29:1-7.
Sakaguchi RL: Review of the current status and challenges for dental posterior restorative composites: clinical, chemistry, and physical behavior considerations. Summary of discussion from the Portland Composites Symposium (POCOS) June 17-19, 2004, Oregon Health and Science University, Portland, Oregon. Dent Mater 2005;21:3-6.

Shi W, Li YH, Liu F, Yang JY, Zhou DH, Chen YQ, Zhang Y, Yang Y, He BX, Han C, Fan MW, Yan HM: Flagellin enhances saliva IgA response and protection of anti-caries DNA vaccine. J Dent Res 2012;91:249-254.

Stock SR, Barss J, Dahl T, Veis A, Almer JD: X-ray absorption microtomography (micro-CT) and small beam diffraction mapping of sea urchin teeth. J Struct Biol 2002;139:1-12.

Stookey GK, Warrick JM, Miller LL, Greene AL: Animal caries models for evaluating fluoride dentifrices. Adv Dent Res 1995;9:198-207; discussion 208-113.

Swain MV, Xue J: State of the art of micro-CT applications in dental research. Int J Oral Sci 2009;1:177-188.

Takahashi N, Nyvad B: The role of bacteria in the caries process: ecological perspectives. J Dent Res 2011;90:294-303.

Taylor AM, Satterthwaite JD, Ellwood RP, Pretty IA: An automated assessment algorithm for micro-CT images of occlusal caries. Surgeon 2010;8:334-340.

Wang SP, Ge Y, Zhou XD, Xu HH, Weir MD Zhang KK, Wang HH, Hannig M, Rupf S, Li $\mathrm{Q}$, Cheng L: Effect of anti-biofilm glass-ionomer cement on Streptococcus mutans biofilms. Int J Oral Sci 2016;8:76-83.

Xu JS, Cui Y, Liao XM, Tan XB, Cao X: Effect of emodin on the cariogenic properties of Streptococcus mutans and the development of caries in rats. Exp Ther Med 2014;8:1308-1312.

Zhang K, Wang S, Zhou X, Xu HH, Weir MD, Ge Y, Li M, Wang S, Li Y, Xu X, Zheng L, Cheng L: Effect of antibacterial dental adhesive on multispecies biofilms formation. J Dent Res 2015;94:622-629.

Zhou H, Li F, Weir MD, Xu HH: Dental plaque microcosm response to bonding agents containing quaternary ammonium methacrylates with different chain lengths and charge densities. J Dentistry 2013;41:1122-1131.

Zhou H, Weir MD, Antonucci JM, Schumacher GE, Zhou XD, Xu HH: Evaluation of threedimensional biofilms on antibacterial bonding agents containing novel quaternary ammonium methacrylates. Int J Oral Sci 2014;6: 77-86. 